
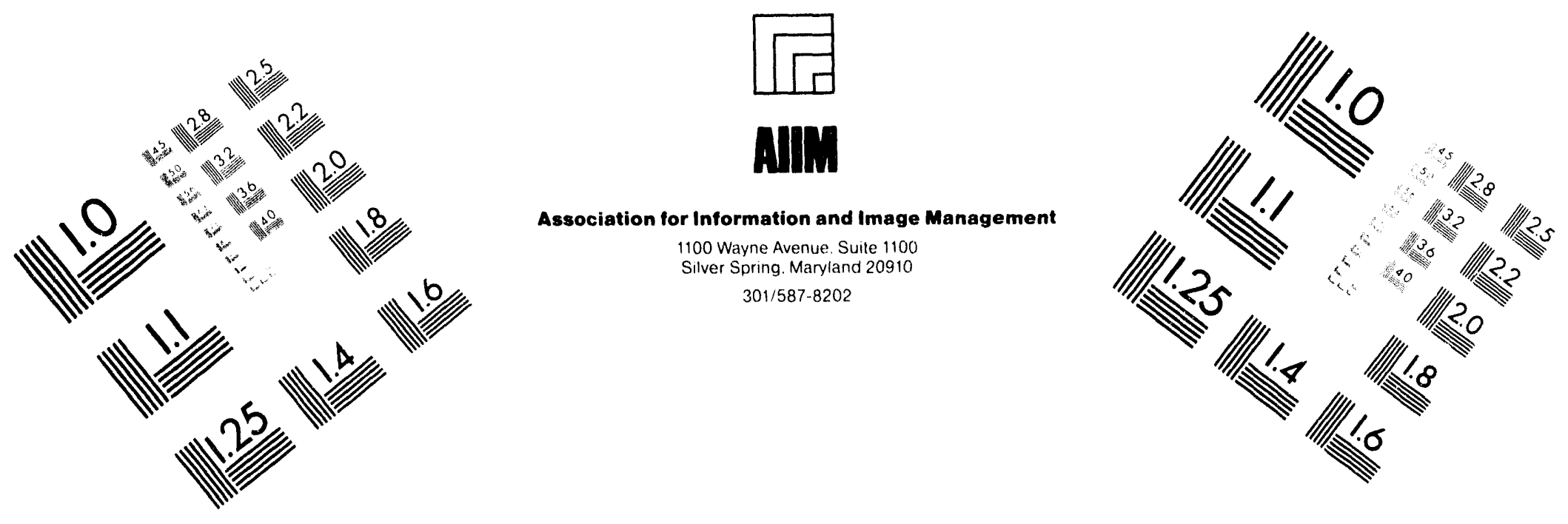

\title{
Centimeter
}
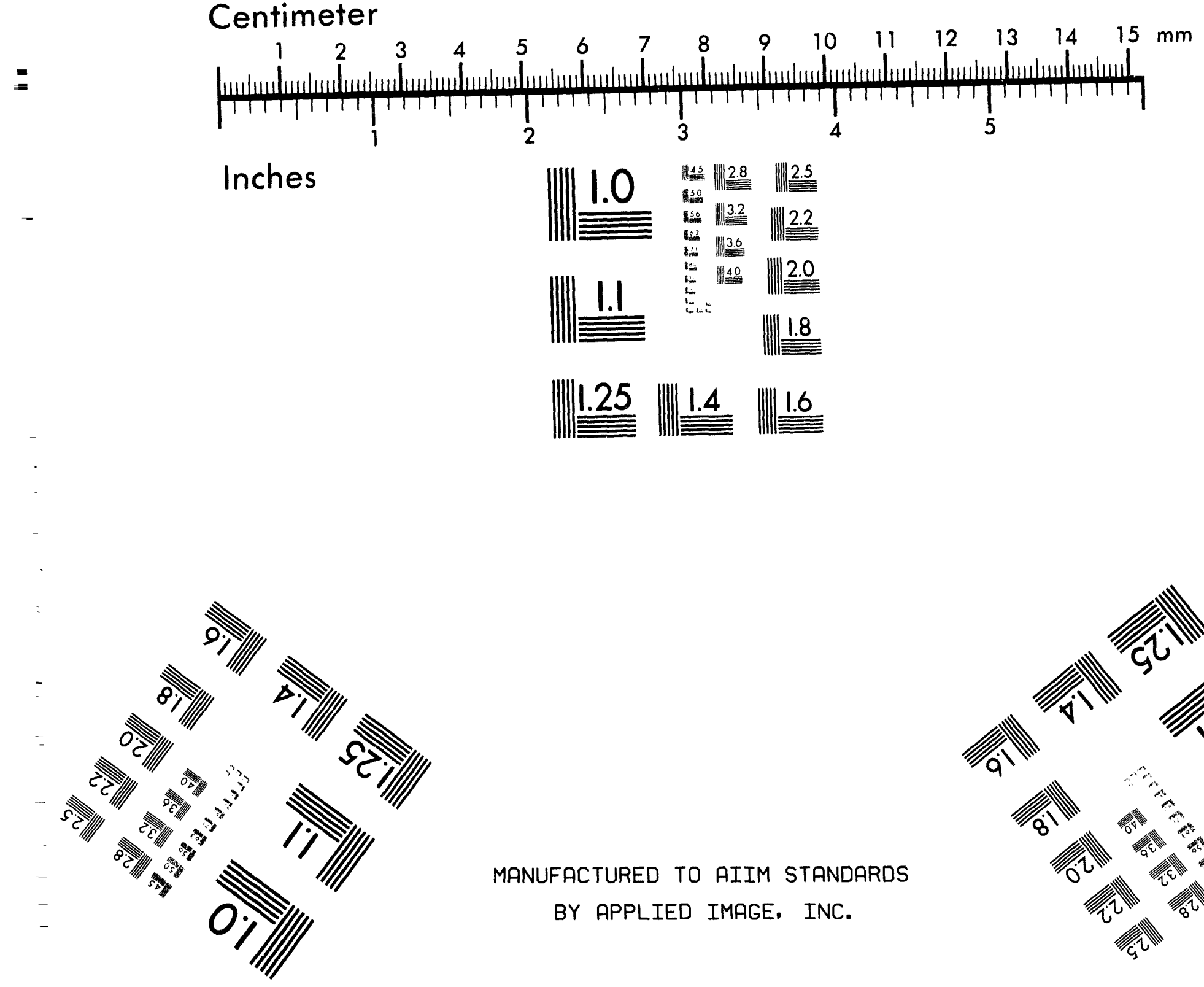

MANUFACTURED TO AIIM STANDARDS

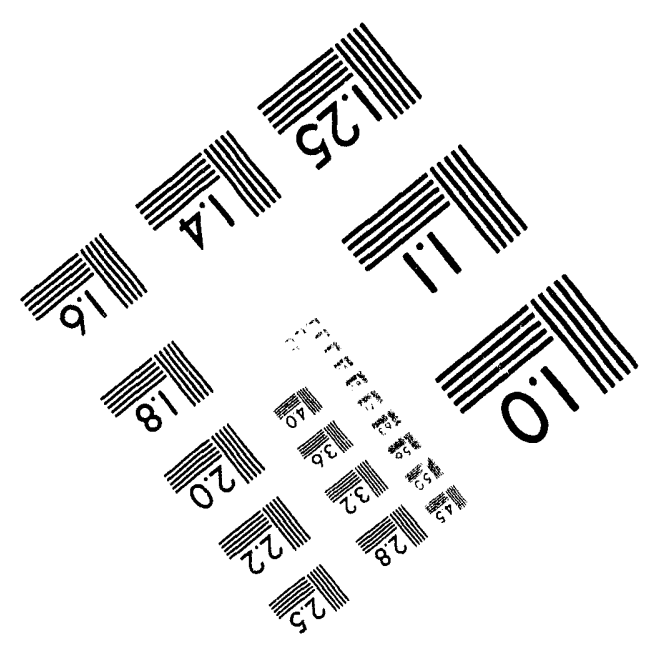



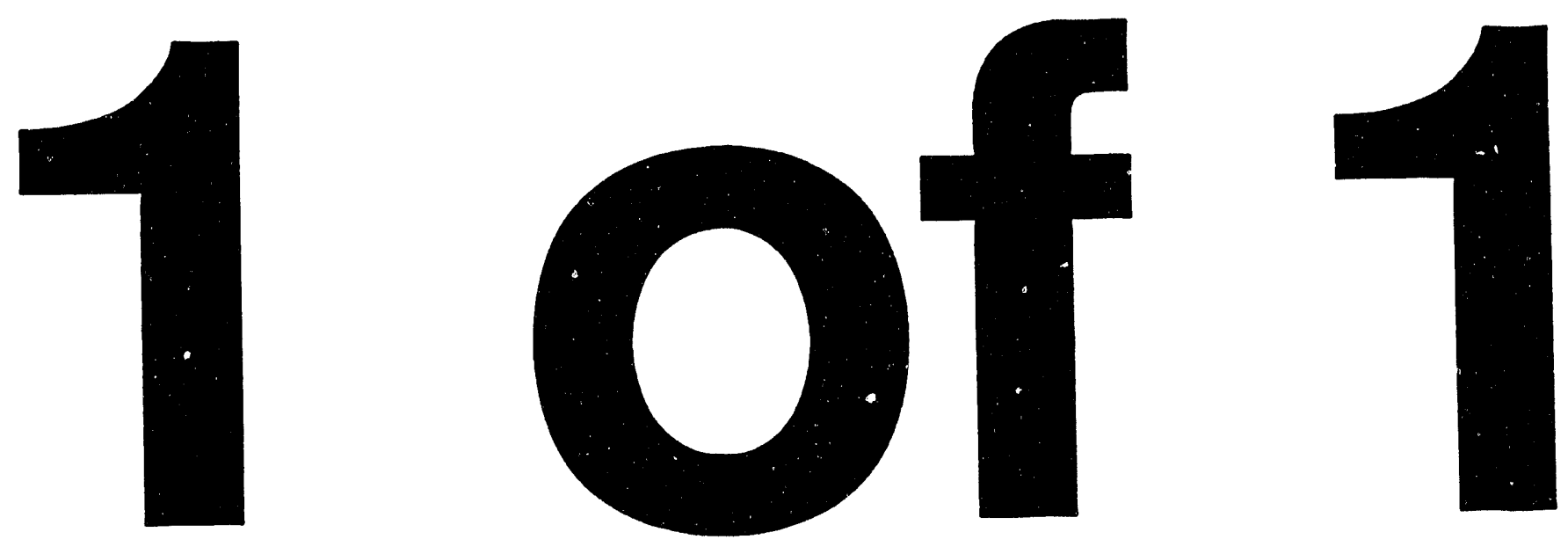


\section{Conf- $940592--6$}

UCRL-JC-1 16926

PREPRINT

\section{Hyperfine Splittings, Prepulse Technique, and other New Results for Collisional Excitation Neon-like X-ray Lasers}

Joseph Nilsen, Juan C. Moreno, Jeffrey A. Koch, James H. Scofield, Brian J. MacGowan, and Luiz B. Da Silva

This paper was prepared for submittal to the Conference Proceedings of 4th International Colloquium on X-ray Lasers

Williamsburg, VA

May 16-20, 1994

June 1994

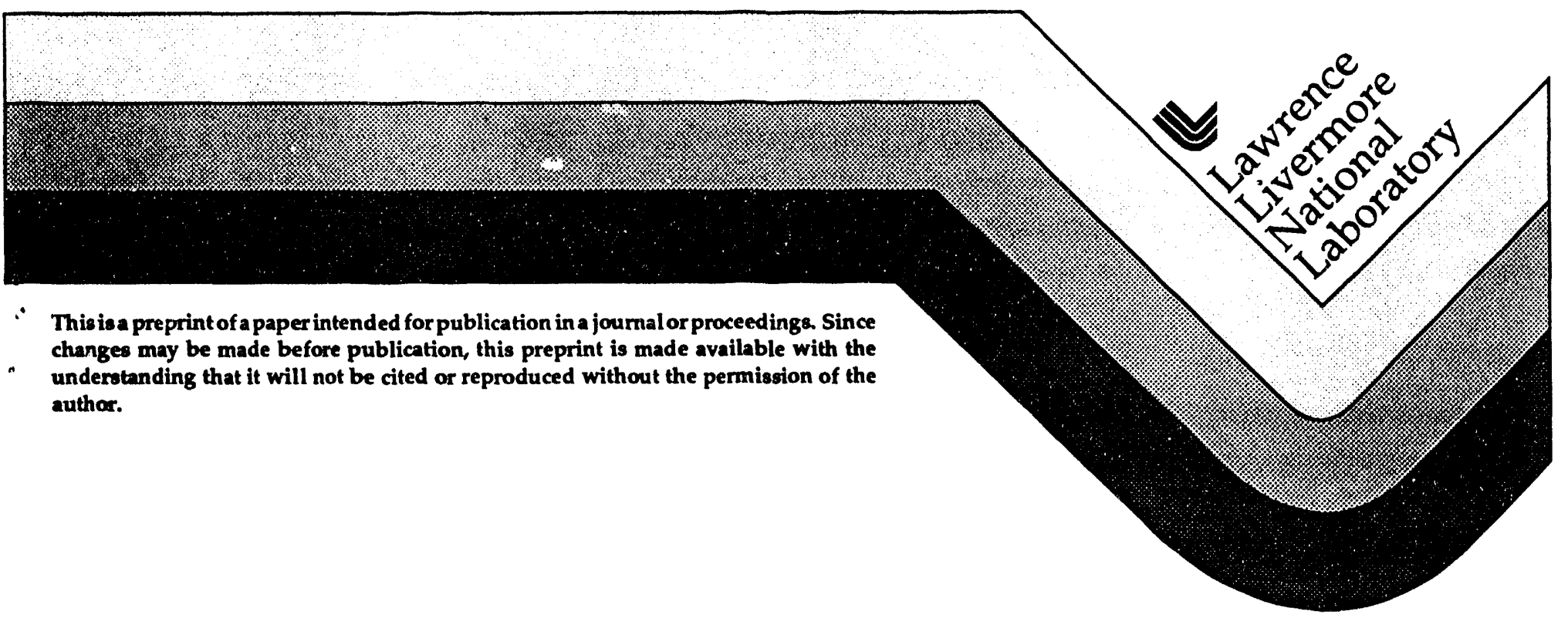




\section{DISCLAIMER}

This document was prepared as an account of work sponsored by an agency of the United States Government. Neither the United States Government nor the University of California nor any of their employees, makes any warranty, express or implied, or assumes any legal liability or responsibility for the accuracy, completeness, or usefulness of any information, apparatus, produch, or process disclosed, or represents that its use would not infringe privately owned rights. Reference herein to any specific commercial products, process, or service by trade name, trademark, manufacturer, or otherwise, does nol necessarily constitute or imply its endorsement, recommendation, or favoring by the United States Government or the University of California. The views and opinions of authors expressed herein do not necessarily state or reflect those of the United States Government or the University of California, and shall not be used for advertising or product endorsement purposes. 


\title{
Hyperfine Splittings, Prepulse Technique, and other New Results for Collisional Excitation Neon-like X-ray Lasers
}

\author{
Joseph Nilsen, Juan C. Moreno, Jeffrey A. Koch, James H. Scofield, \\ Brian J. MacGowan, and Luiz B. Da Silva
}

Lawrence Livermore National Laboratory, P. O. Box 808, Livermore, California 94550

\begin{abstract}
The observation of hyperfine splitting on an $\mathrm{X}$-ray laser transition is presented and the impact on the laser gain is discussed. We measure the lineshape of the $3 p \rightarrow 3 s(J=$ $0 \rightarrow 1$ ) transition in neon-like niobium and zirconium and observe a $28 \mathrm{~m} \AA$ splitting between the two largest hyperfine components in the niobium $(Z=41)$ line at $145.9 \AA$, in good agreement with theory. In zirconium $(Z=40)$, no splitting is predicted or observed since the hyperfine effect is proportional to the nuclear moment and this is present primarily in elements with odd $Z$. The hyperfine splitting is used to explain why the low- $Z$ ions with odd $Z$ have not lased. We discuss the use of a prepulse technique to achieve lasing in low- $Z$ neon-like ions from $Z=22$ to 32 on the $3 p \rightarrow 3 s(J=0 \rightarrow 1)$ transition with wavelengths from 326 to $196 \AA$. Using this technique on selenium $(Z=34)$ we show a large enhancement of the $\mathrm{J}=0 \rightarrow 1$ transition at $182 \AA$. Using a series of short pulses to drive selenium we observe the $182 \AA$ line to completely dominate the spectra. In an effort to reduce the large density gradients associated with hydrodynamic expansion, we discuss the use of low density foams for the laser target and present results which show lasing in zirconium aerogel with an initial density of $20 \mathrm{mg} / \mathrm{cm}^{3}$. Finally we discuss recent double slab experiments with ruthenium $(Z=44)$ targets in which we observe lasing at 117 and $118 \AA$ for the first time.
\end{abstract}

\section{Experimental Setup}

Experiments were conducted at Lawrence Livermore National Laboratory (LLNL) on the Nova laser using $\lambda=0.53 \mu \mathrm{m}$. In a typical experiment on the low- $\mathrm{Z}$ materials the Nova laser illuminates a $125 \mu \mathrm{m}$ thick, $4.5 \mathrm{~cm}$ long slab target of nickel. The actual target length is reduced to $3.8 \mathrm{~cm}$ by a $16 \%$ gap in the center. The pump laser beam was a $600 \mathrm{ps} \mathrm{FWHM} \mathrm{gaussian} \mathrm{pulse} \mathrm{with} 1100 \mathrm{~J}$ of energy in a $120 \mu \mathrm{m}$ wide (FWHM) by $5.4 \mathrm{~cm}$ long line focus, resulting in a peak intensity of $34 \mathrm{TW} / \mathrm{cm}^{2}$. A $6 \mathrm{~J}$ prepulse (also $600 \mathrm{ps}$ FWHM) preceded the main pulse by $7 \mathrm{~ns}$. For the selenium experiments, a $1 \mu$ thick coating of selenium on a nickel substrate was used as the target and the main pulse was increased to $2200 \mathrm{~J}$ while keeping the prepulse energy and delay the same as above. As an alternative to using a weak prepulse before the main pulse, we also illuminated targets with a 
series of three 100 ps FWHM gaussian pulses which were 400 ps apart and had 250 to $400 \mathrm{~J}$ of energy in each pulse. For the niobium and zirconium experiments $3.0 \mathrm{~cm}$ long slab targets were illuminated by a 500 ps square pulse with $2.4 \mathrm{~kJ}$ of energy in a $120 \mu \mathrm{m}$ wide (FWHM) by $3.6 \mathrm{~cm}$ long line focus, resulting in a peak intensity of $130 \mathrm{TW} / \mathrm{cm}^{2}$. For the zirconium aerogel experiment a $2.0 \mathrm{~cm}$ long target was illuminated by a 500 ps square pulse with $2.4 \mathrm{~kJ}$ of energy in a $120 \mu \mathrm{m}$ wide (FWHM) by $2.4 \mathrm{~cm}$ long line focus, resulting in a peak intensity of 200 $\mathrm{TW} / \mathrm{cm}^{2}$ while the ruthenium experiments used $1.0 \mathrm{~cm}$ long targets illuminated with the same beam energy, and therefore twice the intensity, as the aerogel.

The principal instruments were a time-gated, microchannei plate intensified grazing-incidence grating spectrograph(MCPIGS) and a streaked flat field spectrograph(SFFS); both of these instruments observed the axial output of the $\mathrm{X}$-ray laser. The MCPIGS provided angular resolution over $10 \mathrm{mrad}$ near the Xray laser axis, while the SFFS integrated over an angular acceptance of $10 \mathrm{mrad}$. The MCPIGS used a 600 line per $\mathrm{mm}$ grating and had spectral coverage of approximately 150 to $680 \AA$. For the zirconium, niobium, and ruthenium experiments a 1200 line per mm grating was used with spectral coverage of approximately 75 to $340 \AA$. When measuring the laser lineshape the SFFS was replaced with a high-resolution, grazing incidence grating spectrometer which recorded time-integrated but spatially resolved data using a Princeton Instruments camera with a backside illuminated EEV CCD. This spectrometer was centered on the laser axis with an angular acceptance of $12 \mathrm{mrad}$ and had a measured spectral

resolution of 20000 at $146 \AA$ and spectral coverage of $2 \AA$. The angular resolution of all three instruments was perpendicular to the target surface.

\section{Experimental Results and Analysis}

Over the last few years we have observed rather different behavior for neon-like lasers which used materials with even $Z$ as compared with odd $Z$. Since elements with odd $Z$ have a nuclear spin and a nuclear moment and those with even $\mathrm{Z}$ tend to have no nuclear spin, one possible explanation for this anomalous behavior is that hyperfine splitting is playing an important role in the gain of the neon-like laser lines. Hyperfine splitting can affect the gain of the laser line by effectively increasing the linewidth. Since the gain is inversely proportional to linewidth the gain will decrease. If the splitting is large enough, a single line may be split into several weaker lines. The hyperfine effect is largest for the $J=0 \rightarrow 1$ line which dominates the spectra of the low- $Z$ neon-like ions. We did a series of experiments, described in a previous paper(1), to measure the lineshape of the $\mathrm{J}=$ $0 \rightarrow 1$ laser line in neon-like niobium. Niobium was chosen because it has a very 
large nuclear spin, $I=9 / 2$, and a large nuclear moment, $\mu=6.167$, its wavelength is in the range of the high resolution spectrometer which we had available, and it had been observed to lase(2). Figure 1 shows the measured(solid) and calculated(dotted) intensity versus wavelength for the niobium line. Two components are clearly visible with a separation of $28 \mathrm{~m} \AA$, which is very close to the $32 \mathrm{~m} \AA$ prediction given the $7 \mathrm{~m} \AA$ resolution of the spectrometer. This is the shortest wavelength transition and most highly ionized plasma in which the hyperfine effect has been directly observed on a laser transition(1). If we consider vanadium, assuming an ion temperature of $50 \mathrm{eV}$ based on calculations, the hyperfine splitting(3) reduces the gain coefficient of the $J=0 \rightarrow 1$ laser line at $304 \AA$ by $40 \%$. Given a nominal gain coefficient of $2.6 \mathrm{~cm}^{-1}$ for titanium $(4,5)$, this reduces the gain coefficient to $1.6 \mathrm{~cm}^{-1}$ for vanadium. For the $3.8 \mathrm{~cm}$ long targets tried with vanadium, this would make the vanadium fifty times weaker than the titanium. While this is still within the detectable range of the diagnostics, the hyperfine effect appears to play a major role in the non lasing of vanadium and scandium and the poor lasing of the other odd $\mathrm{Z}$ ions(3).

Using the prepulse technique described above we have done experiments(46) on all elements from calcium $(Z=20)$ to germanium $(Z=32)$ with the exception of gallium $(\mathrm{Z}=31)$. Different illumination conditions were used depending on the element but most were tried with the nominal conditions described above. Lasing

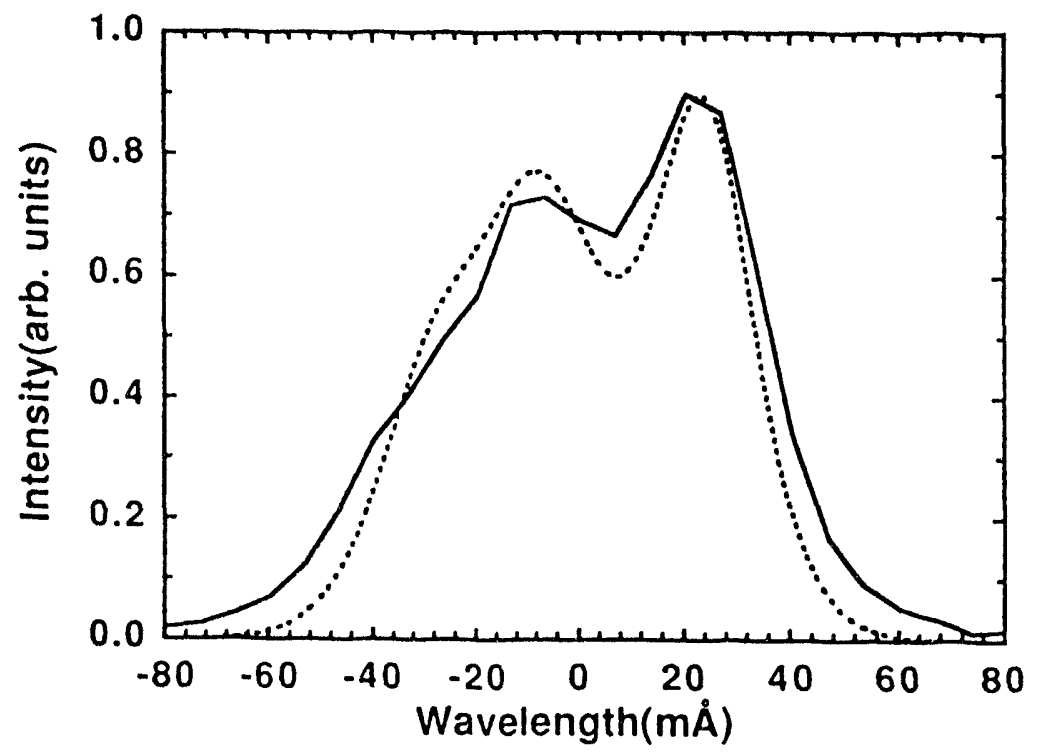

Fig. 1. Measured(solid line) and calculated(dotted line) intensity vs wavelength around line center for the $\mathrm{J}=0 \rightarrow 1$ laser line in neon-like niobium at $145.9 \AA$. The curves are normalized to the same intensity and a peak gain-length product of 2 is assumed for the calculated curve. 
was determined by observing the high spectral brightness of the lasing lines relative to the strong emission lines on-axis, the absence of the lasing lines off-axis, the short time duration of the lasing relative to the optical drive pulse, and the exponential growth of the laser output as the length was increased. In the experiments(4-6) we saw strong lasing on the $3 \mathrm{p} \rightarrow 3 \mathrm{~s}(\mathrm{~J}=0 \rightarrow 1)$ lines at 326, $285,255,231,212$, and $196 \AA$ in titanium, chromium, iron, nickel, zinc, and germanium, respectively while scandium $(Z=21)$, vanadium $(Z=23)$, and manganese $(\mathrm{Z}=25)$ do not lase. Weak lasing was observed in cobalt $(\mathrm{Z}=27)$ and copper $(Z=29)$. Calcium did not lase but we attribute that to target handling difficulties. For the even $\mathrm{Z}$ ions we also observed some weak $\mathrm{J}=2 \rightarrow 1$ laser lines. Figure 2 shows the spectrum from the MCPIGS spectrograph for a zinc target. The strong $\mathrm{J}=0 \rightarrow 1$ laser line at $212 \AA$ completely dominates the spectrum and is fifteen times more intense than the weak $J=2 \rightarrow 1$ laser lines at 262 and $267 \AA$. In recent experiments(7) on selenium using the prepulse technique, the J $=0 \rightarrow 1$ line at $182 \AA$ suddenly jumps up and becomes a strong line as was originally predicted but never observed in standard $X$-ray laser experiments $(8)$. However, unlike the lower- $Z$ cases, the $J=2 \rightarrow 1$ lines at 206 and $209 \AA$ still dominate the spectrum. Based on our calculations $(4,5)$, we believe the prepulse is playing a key role in creating a larger, more uniform density plasma, at the densities required for lasing at these wavelengths. The combination of the small gain region with the inability to propagate the length of the laser is no doubt the reason the low- $\mathrm{Z}$ neon-like lasers have not worked without the prepulse.

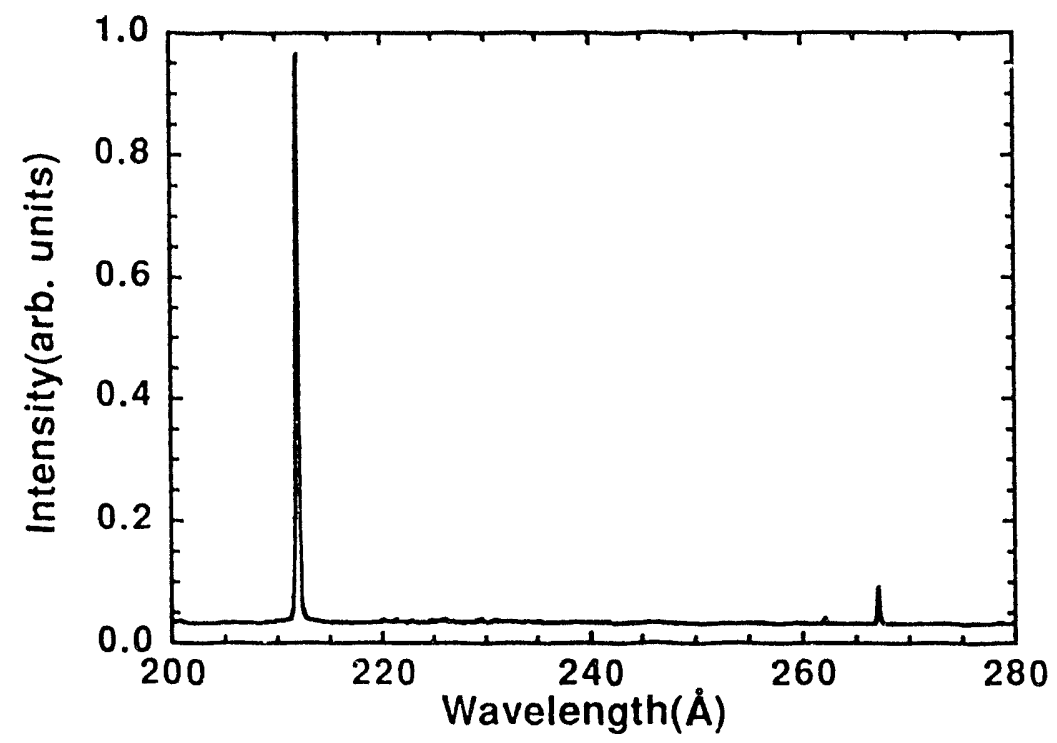

Fig. 2. MCPIGS spectrum of a $3.8 \mathrm{~cm}$ long target of zinc using the prepulse technique. 
As another approach to preforming a plasma, we have done experiments on germanium and selenium targets using a multiple pulse technique. For the selenium experiments we illuminated $3.0 \mathrm{~cm}$ long targets with a series of three $100 \mathrm{ps}$ FWHM gaussian pulses which were $400 \mathrm{ps}$ apart and had $400 \mathrm{~J}$ of energy in each pulse. Figure 3 shows the selenium spectrum from the MCPIGS spectrograph. The $182 \AA$ line completely dominates the output. The time resolved spectrum shows strong lasing on the second and third pulse. The first pulse provides the initial heating and expansion of the plasma, thereby preparing the plasma for lasing during the subsequent pulses.

To eliminate the violent hydrodynamics which takes place when a solid target is heated by an optical laser such as Nova we are pursuing the use of foam targets which could potentially be fabricated at the final density needed for lasing and be volume heated by the Nova laser, thereby eliminating the large density gradients in the plasma. These density gradients cause significant refraction of the X-ray laser as it propagates down the laser axis and limits the effective length of the plasma as well as the laser coherence. We have tried several experiments on different foams with modest success. To achieve very low density we have tried molybdenum-doped agar foam (a hydrocarbon foam) at $3 \mathrm{mg} / \mathrm{cm}^{3}$ and selenium-doped agar foam at $8 \mathrm{mg} / \mathrm{cm}^{3}$. Both foams were nominally $50 \%$ metal by weight and neither lased. The agar has the difficulty that the cell size is micron scale and the foam must be doped with the element of interest, either as small particles or a compound. A more promising route is using aerogels such as

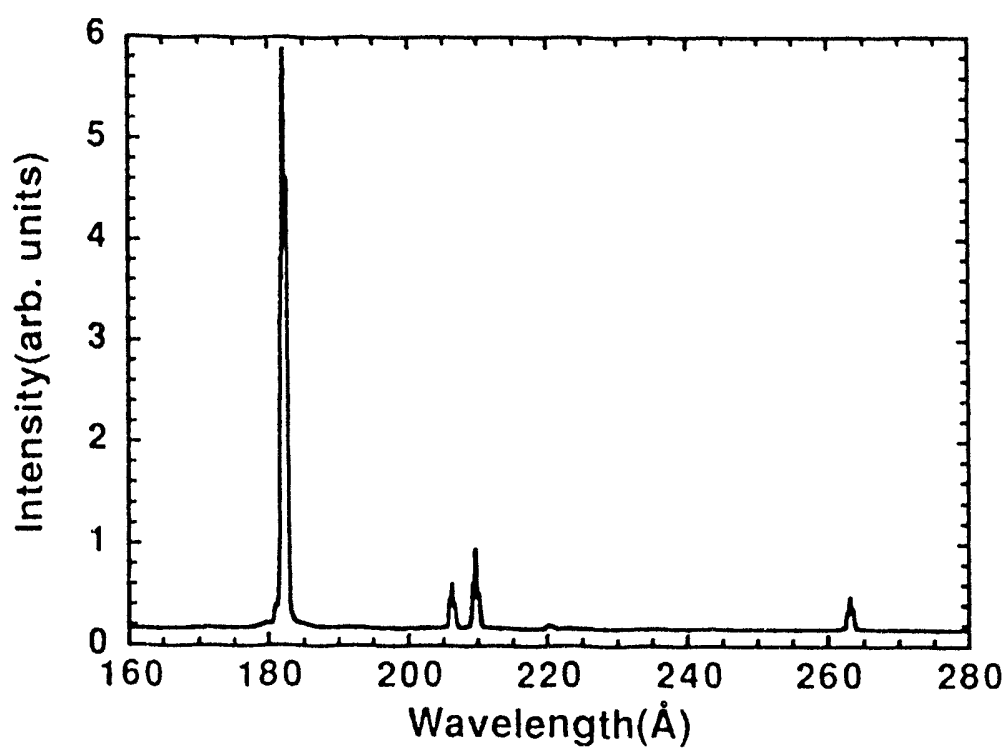

Fig. 3. MCPIGS spectrum of a $2.5 \mathrm{~cm}$ long target of selenium illuminated with multiple pulses. 
zirconium aerogel which has a small cell size the order of $500 \AA$ and is pure zirconium oxide, so the doping issue is avoided. We have tried zirconium aerogel with densities of 470,90 , and $20 \mathrm{mg} / \mathrm{cm}^{3}$ and they have lased quite well, as shown in Fig. 4 which presents the intensity versus wavelength as measured with the MCPIGS spectrograph for the lowest density foam. The pair of $J=2 \rightarrow 1$ lines at 146 and $148 \AA$ lase quite well. Presently we are trying to produce lower

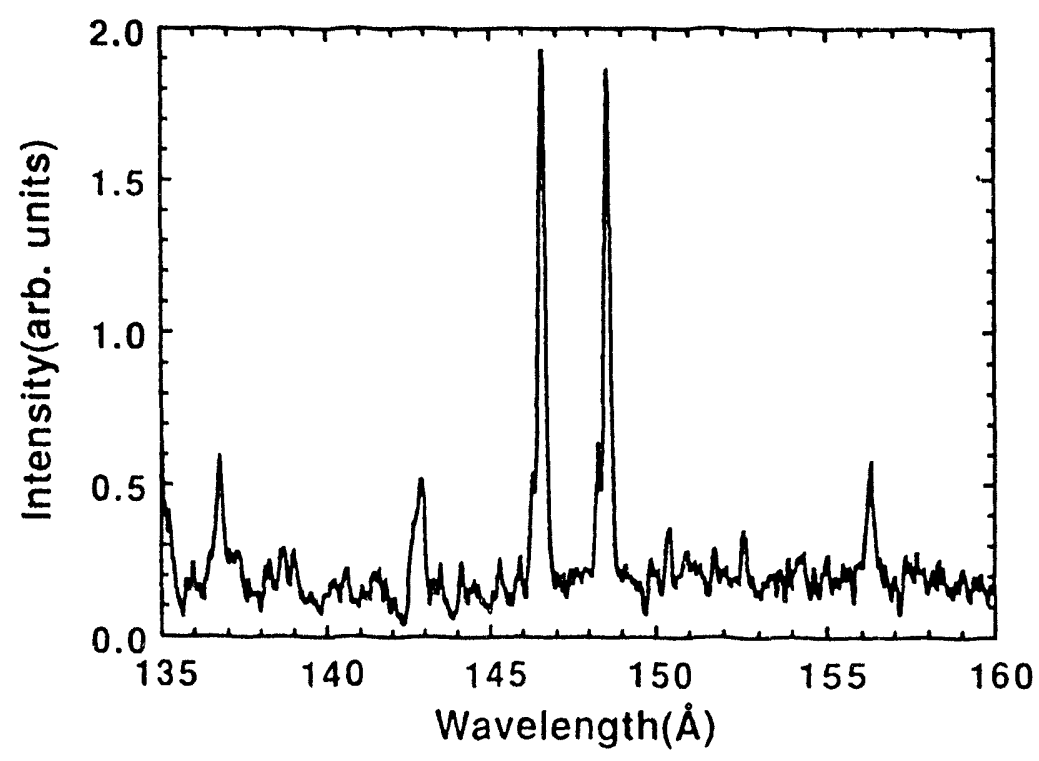

Fig. 4. MCPIGS spectrum of a $1.7 \mathrm{~cm}$ long target of zirconium acrogel.

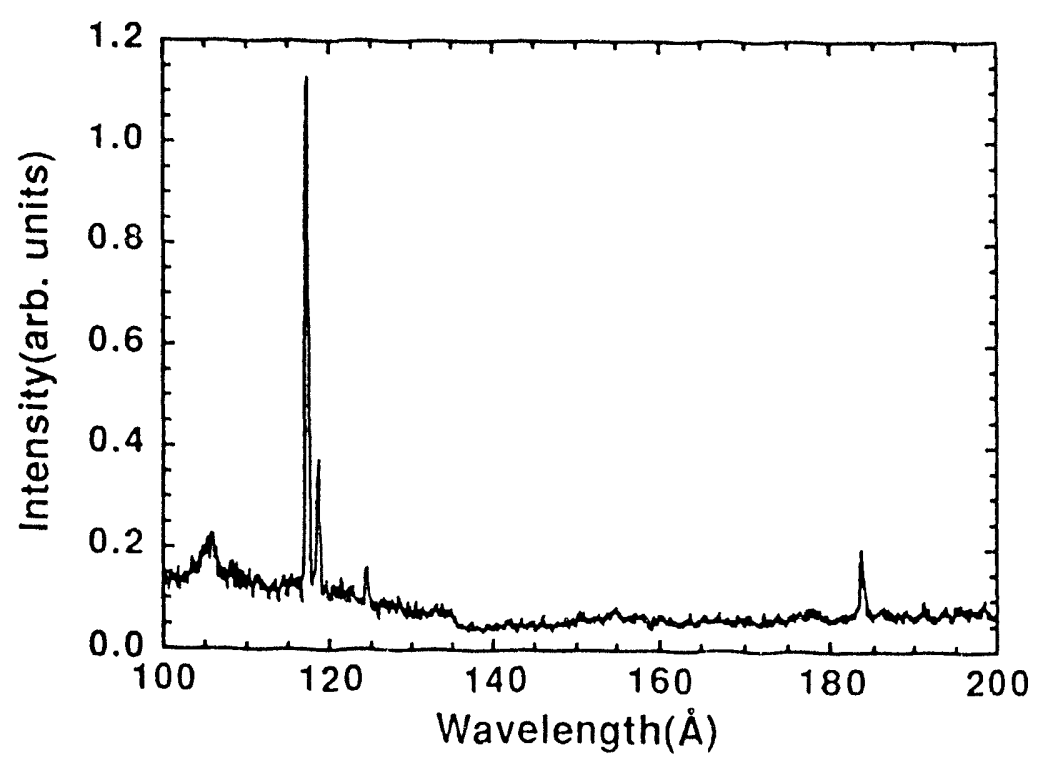

Fig. 5. MCPIGS spectrum of a double slab target of ruthenium. 
density aerogel. Silicon aerogel is the most mature technology and can be produced down to $1 \mathrm{mg} / \mathrm{cm}^{3}$ so we are optimistic that the zirconium aerogel density can be lowered to the $1-3 \mathrm{mg} / \mathrm{cm}^{3}$ range appropriate for lasing.

In an effort to make brighter X-ray lasers with slab targets we have been doing experiments using double slab targets. We recently observed lasing in ruthenium( $(Z=44)$ at 117 and $118 \AA$, as shown in Fig. 5, using this approach. The target consisted of two $1.0 \mathrm{~cm}$ long slab targets which were separated by $300 \mu$ in the transverse dimension and were aligned end to end with a separation of $0.5 \mathrm{~cm}$. The slabs were illuminated from opposite sides by two beams of the Nova laser using $2.4 \mathrm{~kJ}$ of energy from each beam in a $500 \mathrm{ps}$ square pulse.

\section{Conclusions}

We show that using the prepulse technique many low- $\mathrm{Z}$ neon-like ions from titanium to germanium lase well on the $3 p \rightarrow 3 s(J=0 \rightarrow 1)$ transition. Using this technique on selenium caused the "missing" J $=0 \rightarrow 1$ line at $182 \AA$ to lase quite strongly. New multiple pulse experiments on selenium show the $182 \AA$ line completely dominating the spectrum. The hyperfine effect is shown to be the dominant line broadening mechanism for the $3 \mathrm{p} \rightarrow 3 \mathrm{~s}(\mathrm{~J}=0 \rightarrow 1)$ neon-like niobium laser line at $145.9 \AA$. We measured the lineshape of this transition and observed a $28 \mathrm{~m} \AA$ splitting between the two largest hyperfine components, in good agreement with theory. This is the largest hyperfine splittings ever measured on a laser transition. In the effort to produce more uniform plasma, lasing is observed for the first time using a foam target of zirconium aerogel. Finally, the double slab target was used successfully to demonstrate lasing in ruthenium at 117 and $118 \AA$ for the first time.

\section{Acknowledgements}

The authors would like to thank Larry Hrubesh for providing the zirconium aerogel and Sharon Alvarez, Hedley Louis, Tony Demiris, Judy Ticehurst, and the Nova facilities crew for providing support for the experiments. The support of S. B. Libby, D. A. Nowak and D. L. Matthews is greatly appreciated. Work performed under the auspices of the U. S. Department of Energy by the Lawrence Livermore National Laboratory under contract No. W-7405-ENG-48.

\section{References}

1. J. Nilsen, J. A. Koch, J. H. Scoficld, B. J. MacGowan, J. C. Moreno, and L. B. Da Silva, Phys. Rev. Lett. 70, 3713-3715 (1993). 
2. J. Nilsen, J. L. Porter, B. J. MacGowan, L. B. Da Silva, and J. C. Moreno, J. Phys. B 26, L243-247 (1993).

3. J. H. Scofield and J. Nilsen, Phys. Rev. A A 49, $2381-2388$ (1994).

4. T. Boehly, M. Russotto, R. S. Craxton, R. Epstein, B. Yaakobi, L. B. Da Silva, J. Nilsen, E. A. Chandler, D. J. Fields, B. J. MacGowan, D. L. Matthews, J. H. Scofield, and G. Shimkaveg, Phys. Rev. A 42, 6962-6965 (1990).

5. J. Nilsen, B. J. MacGowan, L. B. Da Silva, and J. C. Moreno, Phys. Rev. A 48, 4682 - 4885 (1993).

6. J. Nilsen, J. C. Moreno, B. J. MacGowan, and J. A. Koch, Applied Physics B 57, 309 - 311 (1993).

7. J. Nilsen and J. C. Moreno, "Using the prepulse technique to enhance the weak $18.2 \mathrm{~nm}$ laser line in neon-like selenium," Opt. Lett. (in press, 1994).

8. R. C. Elton, X-ray Lasers (Academic Press, Inc., San Diego, 1990), pp. 99 - 126. 

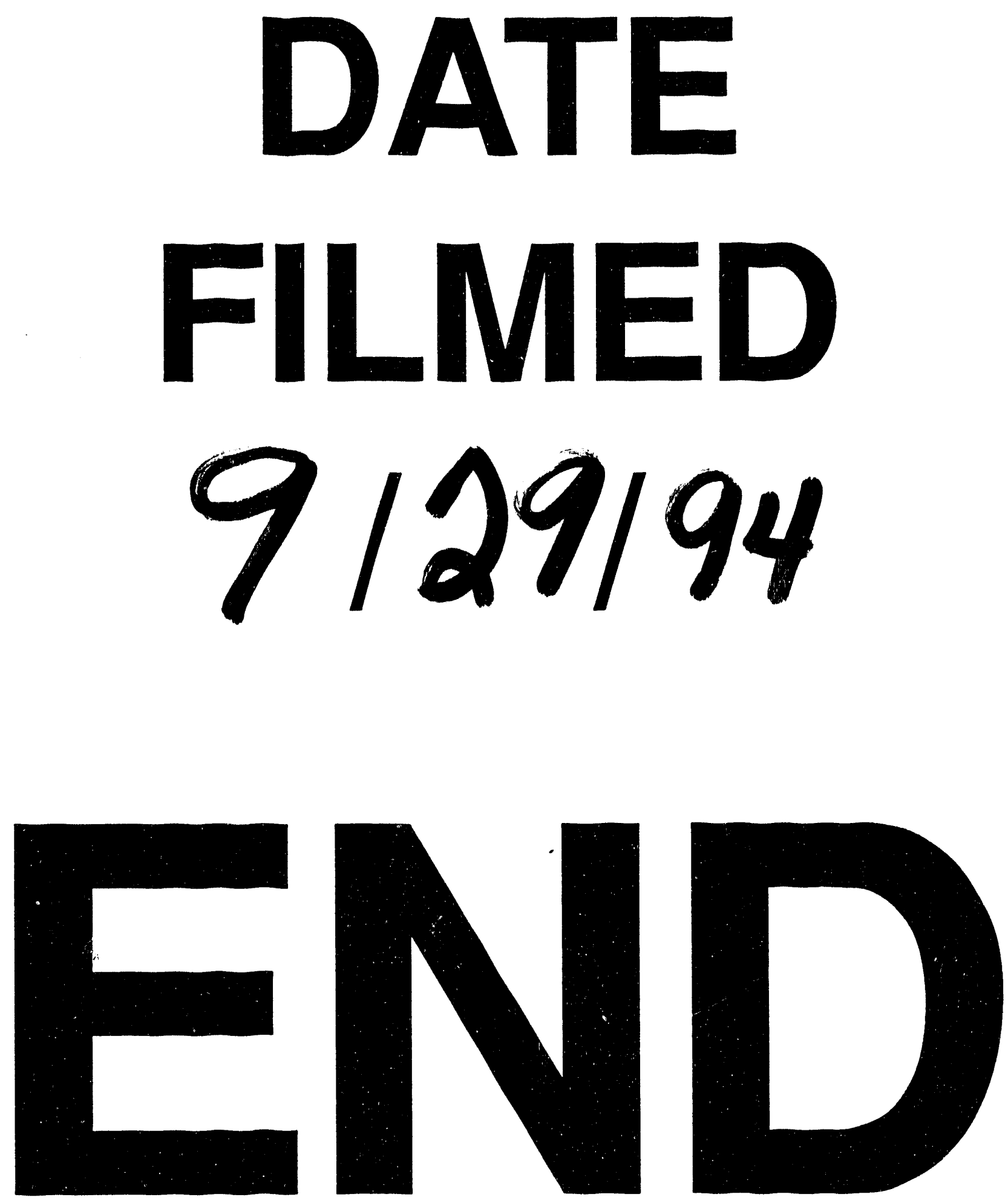